\title{
Patient preferences and expectation for feedback on adverse drug reaction reports submitted in Ghana
}

\author{
George Tsey Sabblah ${ }^{1}$, Delese Darko ${ }^{1}$, Linda Härmark ${ }^{2}$ and Eugène van Puijenbroek ${ }^{2,3}$ \\ Ghana Med J 2019; 53(2): 150-155 doi: http://dx.doi.org/10.4314/gmj.v53i2.10
}

\author{
${ }^{1}$ Food and Drugs Authority, P. O. Box CT2781, Accra, Ghana \\ ${ }^{2}$ Netherlands Pharmacovigilance Centre Lareb, WHO Collaborating Centre for Pharmacovigilance in \\ Education and Patient Reporting, 's-Hertogenbosch, The Netherlands \\ ${ }^{3}$ PharmacoTherapy, Epidemiology and Economics, Groningen Research Institute of Pharmacy University of \\ Groningen, Groningen, The Netherlands
}

Corresponding author: George Tsey Sabblah

E-mail: georgesabblah@gmail.com

Conflict of interest: None Declared

\section{SUMMARY}

Background: Personalized feedback received for spontaneous adverse drug reaction (ADR) reports serves as motivation for future reporting and the effectiveness of the feedback is dependent on the medium used in delivering the information.

Objective: Explore expectation for feedback from patients on ADR reports submitted to the National Pharmacovigilance Centre ( $\mathrm{NPvC})$ in Ghana and the preferred medium for receiving the feedback information.

Methods: Cross-sectional study using structured questionnaire administered through face-to-face interview from August to September 2016 to patients selected by convenience sampling. Pearson chi-square $\left(\mathrm{X}^{2}\right)$ or Fisher's exact test was used to determine associations between background variables such as age, gender and level of education.

Results: The response rate was $86.7 \%(\mathrm{n}=442)$. Of the participants interviewed, $96.5 \%$ expected to receive feedback for ADR reports submitted. Age and level of education were the two variables significantly associated with patients' expectation for feedback.

The preferred medium for receiving feedback in decreasing order of preference were, telephone call (60.4\%), mobile phone short messaging services $(23.0 \%)$, email $(8.3 \%)$, face-to-face meeting $(3.4 \%)$, personalized letter $(3.4 \%)$ and publication in a newsletter (1.4\%).

Conclusion: Patients' expectation for receiving feedback for ADR reports submitted to the NPvC is in line with modern trends in communication. $\mathrm{NPvC}$ should explore these alternatives for providing feedback to patients. This study is limited to what patients' expectations and preferences were for receiving feedback on ADR reports submitted, additional study to further explore the type of information patients expect to be contained in the feedback will be useful to National Pharmacovigilance Centres.

Keywords: Adverse drug reaction, expectation, feedback, patient, preference, spontaneous reporting Funding: None declared

\section{INTRODUCTION}

Spontaneous reporting of adverse drug reactions is one of the major pharmacovigilance methods used for the identification of unknown safety signals of marketed drugs. Providing feedback to reporters on ADR reports submitted in a spontaneous reporting system will serve as motivation for future reporting.

Provision of feedback to reporters has been identified as one of the core process indicators of a functional pharmacovigilance system by the World Health Organization. ${ }^{1}$
Lack of personalized feedback on previous ADR reports submitted to National Pharmacovigilance Centres has been shown to serve as a barrier to future reporting ${ }^{2}$, however, an 11-country survey by van Hunsel et al. revealed that only four countries out of the eleven surveyed send such feedbacks to patients on ADR reports submitted. ${ }^{3}$

Studies which evaluated expectation for feedback from patients on ADR reports submitted discovered that 32.9\% to $60.6 \%$ anticipated receiving feedback after submitting ADR report to National Pharmacovigilance Centres. ${ }^{4,5}$ 
National Pharmacovigilance Centres have provided feedback to reporters in the form of personalized letters and emails $^{3}$, however, to the best of our knowledge, there is no information in the literature at the moment regarding the preferred medium for receiving feedback by consumers and patients. It is important to note that in providing feedback, the choice of the wrong medium can act as a barrier to delivering the desired information and prevent the receiver from taking notice of the message.

In Ghana, the National Pharmacovigilance Centre has always provided feedback to healthcare professionals who submitted ADR reports in the form of personalized letters addressed to each individual reporter. With the introduction of patient reporting initiative in June $2016^{6}$, patients are expected to submit ADR reports to the NPvC. The need therefore to provide feedback to patients on reports received is very important.

Telephone calls, email, short messaging services (SMS) and letters have been used as reminders to patients few days prior to attending doctor's appointment with high success. ${ }^{7,8}$ In Sub-Saharan Africa, mobile technology have been used for several health interventions including adherence to anti-retroviral and antimalarial treatments. ${ }^{9-}$ 11

This study is needed first of all to help National Pharmacovigilance Centres design a specific feedback delivering method for ADR reports submitted by patients because a one-size-fits-all feedback delivery method cannot satisfy different categories of patients and secondly to gather knowledge on patients' expectation for feedback in a low- and middle-income setting. Providing feedback information to reporters will be helpful in improving knowledge of patients on the safety of medicines and also contribute to the quality of reports as suggested by Oosterhuis et. al. ${ }^{12}$

The primary objective of this study is to explore expectations for feedback from patients on ADR reports submitted to the Ghana National Pharmacovigilance Centre. The secondary objectives are to determine the preferred medium for receiving the feedback information and the influence of gender, age and level of education on the choice of the medium.

\section{METHODS}

A cross-sectional survey was carried out from August 25, 2016 to September 30, 2016 by administering a paperbased questionnaire to patients selected by convenience sampling who visited two Pharmacies in two communities in the Ga East Municipal District in the Greater Accra Region and Sekyere South District in the Ashanti Region.

\section{Questionnaire Design}

The questionnaire was designed by the principal investigator and pre-tested with thirty (30) participants who were representative of the target population, but the results are not included in the analysis. The pre-testing was done to assess clarity of wordings of the questions, the question sequence and the possibility of using the questionnaire in the study setting.

The questionnaire consisted of three sections (I to III), namely demographic information, expectations for feedback and the preferred medium for receiving the feedback information. Section I includes questions such as respondents' gender, age, the level of education and employment status, Section II asked whether participants expected feedback for ADR reports submitted to the National Pharmacovigilance Centre and Section III relates to the preferred medium for receiving the feedback information.

To ensure that participants were not interviewed more than once, the names were entered on a separate MS Excel Sheet by the research assistants and deleted after checking for possible duplicates.

\section{Study Population and Exclusion Criteria}

Participants were 18 years and above and selected by convenience sampling from patients dispensed with either prescription, pharmacist-initiated or over-the-counter medicines in two community pharmacies from $\mathrm{Ga}$ East Municipal District in the Greater Accra Region and Sekyere South District in the Ashanti Region. The two community pharmacies were selected such that the Greater Accra region represented an urban population and the Ashanti region rural population. The average number of patients visiting the two pharmacies during the period of the study was between 80 to 120 patients per day. Healthcare professionals (doctors, pharmacists, nurses, midwives and laboratory staff) were excluded from this study to avoid bias. The questionnaire was translated into the local language by the research assistants to participants who did not understand English. Those who agreed to take part in the study signed a consent form to do so and the questionnaire was administered through a face-to-face interview by two research assistants trained by the principal investigator.

\section{Data analysis}

Descriptive statistics was used to describe the demographic characteristics of the participants, expectation for feedback and the preferred medium for receiving feedback. Pearson chi-square $\left(X^{2}\right)$ or Fisher's exact test was used to determine associations between patient's characteristics such as age, gender, level of education, employment status and responses about expectation for feedback 
and the preferred medium for receiving the feedback. Data collected during the study was analyzed using STATA, version 13 by Stata Corp (Texas 77845 USA).

\section{Ethical Consideration}

Ethical approval for the study was granted by the Committee on Human Research, Publications and Ethics (CHRPE), Kwame Nkrumah University of Science and Technology, School of Medical Sciences and Komfo Anokye Teaching Hospital with reference No. CHRPE/AP/380/16. No identifiers of the participants were provided on the questionnaire except signatures, initials or thumbprint, which showed their consent to participate in the study.

\section{RESULTS}

A total of 510 participants were approached in the two study locations and 442 agreed to take part in the interview representing a response rate of $86.7 \%$. Of the completed questionnaires, 8 were excluded from the analysis because of incomplete information.

Table 1 Characteristics of respondents

\begin{tabular}{|l|l|l|}
\hline Variable & $\begin{array}{l}\text { Frequency } \\
\text { (Percent) }\end{array}$ & $\begin{array}{l}\text { Characteristics } \\
\text { Adult Ghanaian }{ }^{\text {a }} \text { (\%) }\end{array}$ \\
\hline Gender & $210(48.4)$ & 47.9 \\
\hline Male & $224(51.6)$ & 52.1 \\
\hline Female & & \\
\hline Age (years) & $180(41.5)$ & 23.1 \\
\hline $\mathbf{1 8 - 2 9}$ & $103(23.7)$ & 12.1 \\
\hline $\mathbf{3 0 - 3 9}$ & $64(14.8)$ & 8.9 \\
\hline $\mathbf{4 0 - 4 9}$ & $45(10.4)$ & 6.9 \\
\hline $\mathbf{5 0 - 5 9}$ & $42(9.7)$ & 7.6 \\
\hline 60+ & & \\
\hline Level of Education & $18(4.2)$ & 22.1 \\
\hline None & $44(10.1)$ & 36.7 \\
\hline Primary & $130(30.0)$ & 44.3 \\
\hline Middle/Junior Secondary & $127(29.3)$ & 45.6 \\
\hline Senior Secondary School & $115(26.5)$ & 3.7 \\
\hline University and above & & \\
\hline Region & $225(51.8)$ & \\
\hline Greater Accra & $209(48.2)$ & \\
\hline Ashanti & & \\
\hline Employment Status & $57(13.11)$ & 48.6 \\
\hline Unemployed & $54(12.4)$ & \\
\hline Student & $169(38.9)$ & \\
\hline Self-employed & $42(9.7)$ & \\
\hline Government employee & $97(22.4)$ & \\
\hline Private sector & $15(3.5)$ & \\
\hline Retired & & \\
\hline & & \\
\hline
\end{tabular}

The characteristics of the 434 patients analyzed are given in Table 1. The median age of the respondents in this study was 36.4 years and the range was 18 to 76 years.

\section{Expectation for feedback}

Almost all participants interviewed, 419(96.5\%), stated that they expect to receive feedback for every report submitted to the National Pharmacovigilance Centre. There was no significant association between expectation for feedback and the participants' region, gender and employment status. Age and level of education, however, were significantly associated with participants' expectation for receiving feedback for adverse drug reactions reports submitted with p-values, as $<0.001$ and 0.001 respectively, (Table 2).

Table 2 Expectation for feedback on reports submitted to the National Pharmacovigilance Centre.

\begin{tabular}{|c|c|c|c|c|}
\hline \multirow[t]{2}{*}{ Characteristic } & \multirow{2}{*}{$\begin{array}{l}\text { Total } \\
\text { No. }\end{array}$} & \multicolumn{2}{|c|}{$\begin{array}{l}\text { Expectation for feed- } \\
\text { back No. }(\%)\end{array}$} & \multirow[t]{2}{*}{$\begin{array}{l}\text { p-value } \\
\left(X^{2}\right)\end{array}$} \\
\hline & & Yes & No & \\
\hline \multicolumn{5}{|l|}{ Region } \\
\hline Greater Accra & 225 & $220(97.8)$ & $5(2.2)$ & \multirow[b]{2}{*}{0.190} \\
\hline Ashanti & 209 & $119(95.2)$ & $10(4.8)$ & \\
\hline \multicolumn{5}{|l|}{ Gender } \\
\hline Male & 210 & $206(98.1)$ & $4(1.9)$ & \multirow[b]{2}{*}{0.115} \\
\hline Female & 224 & $213(95.1)$ & 11(4.9) & \\
\hline \multicolumn{4}{|l|}{ Age (years) } & \multirow{6}{*}{$<0.001$} \\
\hline $18-29$ & 180 & $177(98.3)$ & $3(1.7)$ & \\
\hline 30-39 & 103 & $101(98.1)$ & $2(1.9)$ & \\
\hline $40-49$ & 64 & $64(100.0)$ & $0(0.0)$ & \\
\hline $50-59$ & 45 & $43(95.6)$ & $2(4.4)$ & \\
\hline $60+$ & 42 & $34(80.9)$ & $8(19.1)$ & \\
\hline \multicolumn{4}{|l|}{ Level of education } & \multirow{6}{*}{0.001} \\
\hline None & 18 & $17(94.4)$ & $1(5.6)$ & \\
\hline Primary & 44 & $37(84.1)$ & $7(15.9)$ & \\
\hline Middle/Junior Secondary & 130 & $126(96.9)$ & $4(3.1)$ & \\
\hline Senior Secondary & 127 & $125(98.4)$ & $2(1.6)$ & \\
\hline University and above & 115 & $114(99.1)$ & $1(0.9)$ & \\
\hline \multicolumn{4}{|l|}{ Employment Status } & \multirow{7}{*}{0.052} \\
\hline Unemployed & 57 & $51(89.5)$ & $6(10.5)$ & \\
\hline Student & 54 & $54(100.0)$ & $0(0.0)$ & \\
\hline Self employed & 169 & $164(97.0)$ & $5(3.0)$ & \\
\hline Private Sector & 42 & $41(97.6)$ & $1(2.4)$ & \\
\hline Government employee & 97 & $95(97.9)$ & $2(2.1)$ & \\
\hline Retired & 15 & $14(93.3)$ & $1(6.7)$ & \\
\hline
\end{tabular}

More participants in early adulthood (18-39 years) and middle adulthood (40-59 years) expected to receive feedback compared to older participants (60 years and above). Participants with higher level of education (Middle/Junior Secondary and above) expected more feedback; $98.1 \%$ compared to those who had no education or primary education, $87.1 \%$.

\section{Preferred medium for receiving feedback}

The most preferred medium for receiving feedback was telephone call to the reporter 262(60.4\%) from the $\mathrm{NPvC}$, followed by SMS 100(23.0\%), others are through email $36(8.3 \%)$, face-to-face meeting $15(3.4 \%)$, personalized letter $15(3.4 \%)$ and the least being publication of the reported information in a newsletter 6(1.4\%), Figure 1. 


\section{Original Article}

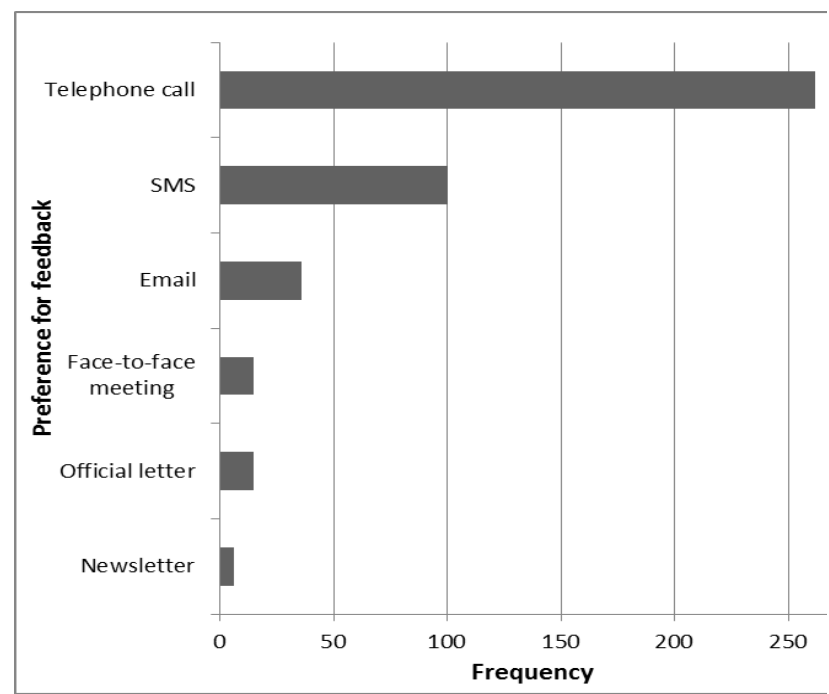

Figure 1 Preferred medium for receiving feedback from the National Pharmacovigilance Centre.

\section{DISCUSSION}

The study resulted in a high response rate of $86.7 \%$ with the percentage of males and females interviewed being $48.4 \%$ and $51.6 \%$ respectively. This is similar to the data obtained during the most recent Population and Housing Census in Ghana for which the percentage of males and females were $48.8 \%$ and $51.2 \%$ respectively ${ }^{14}$. There were, however, differences between the study population with respect to age group, level of education and employment status as compared to the adult Ghanaian population based on the data from the 2010 population and housing census. These differences may be due to the fact that the study sample was obtained from patients who visited community pharmacies and not from the entire adult population in Ghana.

\section{Expectation for feedback}

A high proportion of participants $(96.5 \%)$ expected to receive feedback from the $\mathrm{NPvC}$ for adverse drug reaction reports submitted. The percentage of patients in our study who expected to receive feedback for adverse drug reaction reports submitted is greater than what have been reported by studies in the United Kingdom and Japan where $32.9 \%$ and $60.6 \%$ of the respondents respectively expected to receive feedback for reports submitted. 4,5

The extremely high percentage of respondents in our study who expected to receive feedback for reports submitted could be due to two types of reporting bias, namely, social desirability bias and obsequiousness bias. ${ }^{15}$ In social desirability and obsequiousness biases the respondents tend to provide answers they feel are socially acceptable because they do not want to disappoint the researcher.
Greater number of younger participants expected to receive feedback compared to older participants; this could be due to the fact that older participants are less demanding with respect to expectation for feedback compared to younger ones.

\section{Preferred medium for receiving feedback}

The two most preferred mediums for receiving feedback were telephone calls and SMS; this finding is significant because a recent World Bank report estimated that nearly $73 \%$ of Africans own a mobile phone ${ }^{16}$. Considering mobile phone as the medium for delivering feedback information will therefore be most helpful. Studies on patients' preference for delivering reminders on scheduled clinic visits also revealed telephone calls and SMS as the two topmost preferred means of sending reminders to patients $8,17,18$; these results are consistent with our study.

The reasons for the choice of the telephone calls and SMS could be due to the fact that those who participated in the study have used their mobile phones as a means of communication and therefore considered this as the most preferred and the easiest communication method. The results also revealed that, of those who participated in the study, $14.2 \%$ and $1.9 \%$ respectively in the Ga East Municipal District and Sekyere South District preferred email as the medium for receiving their feedback information. The differences in preference for email amongst participants from the two districts may be explained by the differences in the information technology infrastructure between urban and rural Ghana.

Mobile phone calls to patients and SMS messaging have been used for health interventions such as adherence to anti-retroviral therapy and antimalarial in Africa with success ${ }^{10,11,19}$. The fact that only $3.4 \%$ of the respondents preferred letters as the means of receiving feedback revealed the general preference for receiving information since the postal service is unpopular in recent years because of new information communication technologies. The top three preferences for participants receiving feedback, namely; telephone call, SMS and email also meant that feedback will be received by patients faster compared to the other methods, namely, face-to-face meeting, personalized letter and newsletter.

This study is limited to what patients' expectations and preferences were for receiving feedback on adverse drug reaction reports submitted to the National Pharmacovigilance Centre. Additional study is needed to explore the type of information patients expect to be contained in the feedback. 


\section{Strengths and Weaknesses}

The study has some weaknesses. First of all, we relied on the voluntary participation of convenient sample of patients which has the potential for selection bias with limited generalizability of the study results to the general population. Random sampling would have been the preferred method to enhance generalizability of the results to the entire population; this method was not employed because the complete and up-to-date list of members of the population from which the random sample will be drawn was not available to the researchers. This study, however, provides the preliminary information regarding patients' preferred medium for receiving feedback on adverse drug reactions and also patients' expectation for receiving feedback in a low to middle-income setting.

Secondly, the two research assistants used for the face to face interviews may unintentionally influence responses by providing additional clarifications to the questions and through verbal and non-verbal cues. This was however, reduced by the training provided by the principal investigator prior to the data collection.

Lastly, the study may be affected by reporting bias (social desirability and obsequiousness biases) because responses by participants could not be verified. ${ }^{15}$ The effect of these on the study outcome was minimized by informing the participants prior to the face-to-face interview that their identities will be kept anonymous.

\section{CONCLUSION}

Patients expect to receive feedback for ADR reports submitted to the National Pharmacovigilance Centre and these expectations are in line with modern trends in communication using new technologies like the mobile phone, short messaging services and email. National Pharmacovigilance Centres should explore these alternatives for providing feedback to patients on adverse drug reaction reports received.

\section{REFERENCES}

1. World Health Organization. WHO pharmacovigilance indicators: a practical manual for the assessment of pharmacovigilance systems. 2015. Available from: http://www.who.int/medicines/areas/quality_safety/safety_efficacy/EMP_PV_Indicators_web_ready_v2.pdf.Accessed March 12, 2017.

2. Mirbaha $\bar{F}$, Shalviri G, Yazdizadeh B, et al. Perceived barriers to reporting adverse drug events in hospitals: a qualitative study using theoretical domains framework approach. Implement Sci. 2015;10:110.
3. van Hunsel F, Härmark L, Pal S, et al. Experiences with adverse drug reaction reporting by patients: an 11-country survey. Drug Saf. 2012;35(1):45-60.

4. Avery AJ, Anderson C, Bond CM, et al. Evaluation of patient reporting of adverse drug reactions to the UK 'Yellow Card Scheme': literature review, descriptive and qualitative analyses, and questionnaire surveys. Health Technol Assess. 2011;15(20):1-234.

5. Yamamoto M, Kubota K, Okazaki M, et al. Patients views and experiences in online reporting adverse drug reactions: findings of a national pilot study in Japan. Patient Prefer Adherence. 2015;9:173-84.

6. Ayamgha B. FDA Launches Patients Safety Programme. Available from: http://www.ghananewsagency.org/health/fda-launches-patients-safety-programme-104704. Accessed July 30, 2016.

7. Macharia WM, Leon G, Rowe BH, et al.. An overview of interventions to improve compliance with appointment keeping for medical services. JAMA. 1992;267(13):1813-7.

8. Wang D, Crilly J, Jaeger L, et al. Assessing patient preferences for delivery of reminders on scheduled visits in a psychiatry ambulatory service. AMIA . Annu Symp proceedings. American Medical Informatics Association. 2007;2007: 776-780

9. Zurovac D, Sudoi RK, Akhwale WS, et al. The effect of mobile phone text-message reminders on Kenyan health workers' adherence to malaria treatment guidelines: a cluster randomised trial. Lancet. 2011;378(9793):795-803.

10. Lester RT, Ritvo P, Mills EJ, et al. Effects of a mobile phone short message service on antiretroviral treatment adherence in Kenya (WelTel Kenya1): a randomised trial. Lancet. 2010;376(9755):1838-45.

11. Pop-Eleches C, Thirumurthy H, Habyarimana JP, et al. Mobile phone technologies improve adherence to antiretroviral treatment in a resource-limited setting: a randomized controlled trial of text message reminders. AIDS. 2011;25(6):825-34.

12. Oosterhuis I, van Hunsel FPAM, van Puijenbroek EP. Expectations for feedback in adverse drug reporting by healthcare professionals in the Netherlands. Drug Saf. 2012;35(3):221-32.

13. Ghana Statistical Service (GSS). Ghana Demographic and Health Survey 2014.; 2015. https://dhsprogram.com/pubs/pdf/fr307/fr307.pdf. Accessed August 4, 2018.

14. Ghana Statistical Service (GSS). Summary report of final results. Available from: http://www.statsghana.gov.gh/docfiles/2010phc/Ce nsus2010_Summary_report_of_final_results.pdf. Accessed April 16, 2017].

15. Grimm P. Social Desirability Bias. Wiley International Encyclopedia of Marketing. 2010, John Wiley \& Sons, Ltd. 
16. World Bank. World Development Report. Available from: $\quad \mathrm{http} / / /$ documents.worldbank.org/curated/en/961621467994698644/pdf/102724-WDRWDR2016Overview-ENGLISH-WebResBox394840B-OUO-9.pdf. Accessed March 13, 2016.

17. Cohen CE, Coyne KM, Mandalia S, et al. Time to use text reminders in genitourinary medicine clinics. Int J STD AIDS. 2008;19(1):12-3.
18. O'Connor MB, Bond U, Saunders JA, et al. The preferences for outpatient clinic appointment reminders among rheumatology patients. JCR J Clin Rheumatol 2009;15(5):258-9.

19. Raifman JRG, Lanthorn HE, Rokicki S, et al. The impact of text message reminders on adherence to antimalarial treatment in Northern Ghana: a randomized trial. PLoS One. 2014;9(10):e109032.

Copyright (C) The Author(s). This is an Open Access article under the CC BY license. 